\title{
Catalogue of anuran types in the Eugenio Izecksohn Herpetological Collection (Amphibia, Anura)
}

\author{
Oswaldo L. Peixoto ${ }^{1} \&$ Marcia dos R. Gomes ${ }^{2}$ \\ ${ }^{1}$ Departamento de Biologia Animal, Instituto de Biologia, Universidade Federal Rural do Rio de Janeiro. 23980-000 \\ Seropédica, Rio de Janeiro, Brasil. E-mail: opeixoto@domain.com.br \\ ${ }^{2}$ Departamento de Zoologia, Instituto de Biologia, Universidade Federal do Rio de Janeiro. Caixa Postal 68044, 21949-900 \\ Rio de Janeiro, Rio de Janeiro, Brasil. E-mail: Ifipegp@hotmail.com
}

\begin{abstract}
A list of type specimens housed at the Eugenio Izecksohn Herpetological Collection is presented, information is given on specimens donation to other herpetological collections, as well as corrections regarding some miss information presented in the original descriptions, also remarks are made on the present conservation status of some specimens.
\end{abstract}

KEY WORDS. Corrections of description data; taxonomy; type list.

RESUMO. Catálogo de tipos de anuros da coleção Eugenio Izecksohn (Amphibia, Anura). Uma lista de exemplares-tipo depositados na Coleção Herpetológica Eugenio Izecksohn é apresentada, informações sobre doação de espécimes a outras coleções herpetológicas são fornecidas, bem como correções de informações apresentadas nas descrições originais, o estado atual de conservação de alguns tipos é comentado.

PALAVRAS-CHAVE. Correções de dados das descrições; lista de tipos; taxonomia.

The Eugenio Izecksohn Herpetological Collection, listed in LeVIngton et al. (1985) under the acronym "EI," was established by 1950 as the core of a research line on frog systematics and natural history. The collection is located in the Departamento de Biologia Animal, Instituto de Biologia, Universidade Federal Rural do Rio de Janeiro, and the senior author, presently the collection curator, should be contacted for loan of specimens. Peixoto \& Izecksohn (2001) reviewed the collection history, and presented some general remarks. Currently the collection houses type material of 55 anuran species, including 32 name bearing specimens. In all, 1246 specimens are primary or secondary types in the collection. Due to historical aspects most samples emphasize southeastern Brazil. The frog fauna in the Collection is specially well-represented for the states of Rio de Janeiro and Espirito Santo. Together they include 90\% of the type material deposited by the staff of the Universidade Federal Rural do Rio de Janeiro. Most of the anuran families of the Brazilian fauna are represented (i.e., Brachycephalidae, Bufonidae, Cyclorhamphidae Hylidae, Hylodidae, Leptodactylidae, Leiuperidae, Microhylidae and Pipidae), but hylids represent 44\% of the types.

Wiley (1981) called attention to the importance of primary and secondary type material and justified their special care. The IczN (1999) stressed the importance of all type material and asked for the publication of type material catalogues by the Institutions that hold zoological collections.
We present, following the general group arrangement of Frost (2007) that include the new aproachs to frogs taxonomy of FaIVOVICH et al. (2005), GRANT et al. (2006) and Frost et al. (2006), a list of all type material deposited in the Eugenio Izecksohn Collection as original designations or not. For each of the types listed, original citation, registration number, type locality, the specimen collection locality, and collectors are given. Dates are presented as day/month/year or month/year. Necessary corrections and some additional remarks are presented regarding material donation to other herpetological collections.

Acronyms used in the text are as follow: (AMNH) American Museum of Natural History; (JJ) Jorge Jim Collection, Universidade do Estado de São Paulo, Botucatu; (MNRJ) Museu Nacional, Universidade Federal do Rio de Janeiro; (KU) Museum of Zoology, Kansas University; (WCAB) Coleção Werner Bokermann, now part of the MZUSP Collection; (MZUSP) Coleção do Museu de Zoologia da Universidade de São Paulo and; (USNM) National Museum of Natural History, Smithsonian Institution.

\section{Brachycephalidae}

Brachycephalus didactylus (lzecksohn, 1971): 2

Original combination: Psyllophryne didactyla Izecksohn.

Type locality: Sacra Família do Tinguá, Paulo de Fronting, State of Rio de Janeiro, Brazil (22 29'S, $\left.43^{\circ} 36^{\prime} \mathrm{W}\right)$, ca $570 \mathrm{~m}$ elev. Holotype: EI 4950, 11/1969, F. C. Rezende, S. T. Albuquerque, E. Izecksohn. 
Paratypes: type locality, EI 4951-84, (EI 4951 as allotype) same data as holotype; EI 4985-5010, 17/12/1969, F. C. Rezende, C. A. G. da Cruz, E. Izecksohn; Reserva Biológica de Tinguá, Nova Iguaçu, State of Rio de Janeiro, Brazil; EI 4190, 03/12/ 1965, J. Jim, S. T. Albuquerque, W. F. Mendonça, A. Lebedenco, E. Izecksohn; EI 4191-92, 10/1967, S. T. Albuquerque, C. A., G. da Cruz, Eugenio Izecksohn; EI 5053-56, 08/4/1970, J. L. B. Araújo, C. A. G. da Cruz, E.Izecksohn.

Remarks: EI 4952 now in JJ; EI 4957 and 4960 now in MZUSP; EI 4958 and EI 4959 now in WCAB; EI 4985 and EI 4986 now in KU.

\section{Eleutherodactylus epipedus Heyer, 1984: 22}

Type locality: vicinities of Parque Nacional de Nova Lombardia, Santa Teresa, State of Espirito Santo, Brazil.

Paratypes: Sitio dos Boza, Santa Teresa, State of Espirito Santo, EI 7294-95, 26/10/1974, S. T. Albuquerque, C. A. G. da Cruz, J. G. da Silva, E. Izecksohn; EI 7296-7300, 1/4/1979, E. Izecksohn, O. L. Peixoto, C. A. G. da Cruz; EI 7301-02, 04, 22/12/1980, E. Izecksohn, P. Cascon, F. C. C. Rocha, O. L. Peixoto; EI 7305-15, 11-12/3/1981, E. Izecksohn, C. A. G. da Cruz, A. A. Reis, S. P. Carvalho e Silva; EI 7316, 3-5/4/1981, C. A. G. da Cruz, E. Izecksohn, S. P. Carvalho e Silva, A. A. Reis, O. L. Peixoto; EI 7318-20, 3-5/4/1981, C. A. G. da Cruz, O. L. Peixoto, S. P. Carvalho e Silva, A. A. Reis, E Izecksohn; EI 7321, 29-30/8/1981, E. Izecksohn, A. L. Carvalho, C. A. G. da Cruz, O. L. Peixoto; EI 7323, 28-29/10/1981, E. Izecksohn, C. A. G. da Cruz, O. L. Peixoto; EI 7326-27, 28-29/10/1981, E. Izecksohn, C. A. G. da Cruz, O. L. Peixoto.

\section{Euparkerella cochranae Izecksohn, 1988: 64}

Type locality: Parque Nacional da Serra dos Orgãos, sub-sede, Guapimirim, Magé, State of Rio de Janeiro, Brazil (22³9'S, $\left.43^{\circ} 02^{\prime} \mathrm{W}\right)$, ca $170 \mathrm{~m}$ elev.

Holotype: EI 7278, 12/03/1982, S. P. Carvalho e Silva, E. Izecksohn.

Paratypes: type locality, EI 7276-7, 02/11/1979, EI 7279, same data as holotype, EI 7280-82, same data as holotype, EI 7699706,08/12/1982, I. Ferreira, A. M. Tannure, E. Izecksohn. Campo dos Escoteiros, Citrolandia, Guapimirim, State of Rio de Janeiro, Brazil, EI 7707-10, 12/04/1984, S. P. Carvalho e Silva, E. Izecksohn, EI 7714-27, 16/03/1982, S. P. Carvalho e Silva.

Remarks: EI 7279 clear and stained, EI 7708/09, not traced, EI 7716/18, not traced, EI 7700/04, now USNM.

\section{Euparkerella robusta Izecksohn, 1988: 67}

Type locality: Mimoso do Sul, State of Espirito Santo, Brazil $\left(21^{\circ} 03^{\prime} \mathrm{S} 41^{\circ} 21^{\prime} \mathrm{W}\right)$, ca $70 \mathrm{~m}$ elev.

Holotype: EI 7283, 04/03/1982, F. S. Carvalho e Silva, S. P. Carvalho e Silva, E. Izecksohn, L. Izecksohn.

Paratypes: EI 1993,17/03/1964, D. Castro, EI 7284-86, same data as holotype.

Remarks: EI 7286 clear and stained, EI 7285, not traced.

\section{Euparkerella tridactyla Izecksohn, 1988: 69}

Type locality: Santa Teresa, State of Espirito Santo, Brazil $\left(19^{\circ} 56^{\prime} \mathrm{S}, 40^{\circ} 36^{\prime} \mathrm{W}\right)$, ca $675 \mathrm{~m}$ elev.

Holotype: EI 7257, 03-05/04/1981, C. A. G. da Cruz, O. L. Peixoto, S. P. Carvalho e Silva, A. A. Reis, E. Izecksohn.

Paratypes: EI 7254-56, 11-12/03/1981, S. P. Carvalho e Silva, A. A. Reis, C. A. G. da Cruz, E. Izecksohn, EI 7258-72, 03-05/ 04/1981, C. A. G. da Cruz, O. L. Peixoto, S. P. Carvalho e Silva, E. Izecksohn.

Remarks: EI 7272 clear and stained, EI 7264/65, now USNM, EI 7259-7261-7269, not traced.

\section{Hylidae: Hylinae}

\section{Aplastodiscus arildae (Cruz \& Peixoto, 1985): 60}

Original combination: Hyla arildae Cruz \& Peixoto.

Type locality: Represa do Guinle, Teresópolis, State of Rio de Janeiro, Brazil $\left(22^{\circ} 24^{\prime} \mathrm{S}, 42^{\circ} 57^{\prime} \mathrm{W}\right)$, ca $870 \mathrm{~m}$ elev.

Holotype: EI 7536, 1/1978, O. L. Peixoto, C. A. G. da Cruz, E. Izecksohn.

Paratypes: type locality, EI 7537, 11/1979, E. Izecksohn, O. L. Peixoto; EI 7538, 2/1980, O. L. Peixoto, C. A. G. da Cruz, E. Izecksohn; EI 7535, 2/1978, Represa dos Guinle, Teresópolis, State of Rio de Janeiro, O. L. Peixoto, C. A. G. da Cruz, E. Izecksohn; EI 913, 9/1959, Itatiaia, State of Rio de Janeiro, E. Gouvêa, S. G. Nunes, E. Izecksohn.

\section{Aplastodiscus cavicola (Cruz \& Peixoto, 1984): 41}

Original combination: Hyla cavicola Cruz \& Peixoto.

Type locality: Santa Teresa, State of Espirito Santo, Brazil (19 $\left.56^{\prime} \mathrm{S}, 40^{\circ} 36^{\prime} \mathrm{W}\right)$, ca $675 \mathrm{~m}$ elev.

Holotype: EI 7341, 29-30/8/1981, A. L. Carvalho, C. A. G. da Cruz, E. Izecksohn, O. L. Peixoto.

Paratypes: type locality, EI 7342-49, same data as holotype; EI 7350-53, 9/1980, E. Izecksohn, I. Ferreira, O. L. Peixoto; EI 7354-56, S. T. Albuquerque, C. A. G. da Cruz, E. Izecksohn. Remarks: EI 7343 now in WCAB; EI 7353 now in MZUSP.

\section{Aplastodiscus flumineus (Cruz \& Peixoto, 1984): 36}

Original combination: Hyla fluminea Cruz \& Peixoto.

Type locality: Parque Nacional da Serra dos Orgãos, Teresópolis, State of Rio de Janeiro, Brazil (22 $\left.24^{\prime} \mathrm{S}, 42^{\circ} 57^{\prime} \mathrm{W}\right)$, ca $870 \mathrm{~m}$ elev.

Holotype: EI 7328, 1/1981, S. F. Reis, O. L. Peixoto, C. A. G. da Cruz, E. Izecksohn.

Paratypes: type locality, EI 7330-32, 2/1980, O. L. Peixoto, C. A. G. da Cruz, E. Izecksohn.

Remarks: EI 7331 now in MZUSP; EI 7332 now in WCAB.

\section{Aplastodiscus leucopygius (Cruz \& Peixoto, 1984): 39}

Original combination: Hyla leucopygia Cruz \& Peixoto.

Type locality: Represa dos Guinle, Teresópolis, State of Rio de Janeiro, Brazil $\left(22^{\circ} 24^{\prime} \mathrm{S}, 42^{\circ} 57^{\prime} \mathrm{W}\right)$, ca $870 \mathrm{~m}$ elev.

Holotype: EI 7333, 22/2/1978, C. A. G. da Cruz, O. L. Peixoto, E. Izecksohn. 
Paratypes: type locality, EI 7334-40, same data as holotype. Petrópolis, State of Rio de Janeiro, EI 3198-99, 1/1965 E. Izecksohn. Paranapiacaba, State of São Paulo, Brazil, EI 1633, 20/1/1963, W. C. A. Bokermann.

\section{Aplastodiscus weygoldti (Cruz \& Peixoto, 1985): 63}

Original combination: Hyla weygoldti Cruz \& Peixoto.

Type locality: Santa Teresa, State of Espirito Santo, Brazil (195 $\left.56^{\prime} \mathrm{S}, 40^{\circ} 36^{\prime} \mathrm{W}\right)$, ca $675 \mathrm{~m}$ elev.

Holotype: EI 7697, 10/1981, P. Weygoldt.

Paratype: type locality, EI 7698, same data as holotype.

\section{Bokermannohyla carvalhoi (Peixoto, 1981): 515}

Original combination: Hyla carvalhoi Peixoto.

Type locality: Parque Nacional da Serra dos Orgãos, Teresópolis, State of Rio de Janeiro, Brazil $\left(22^{\circ} 24^{\prime} \mathrm{S}, 42^{\circ} 57^{\prime} \mathrm{W}\right)$, ca $900 \mathrm{~m}$ elev.

Holotype: EI 5601, 07/11/1979, O. L. Peixoto, J. P. Pimentel, E. Izecksohn.

Paratypes: type locality, EI 5602, same data as holotype; EI 5603, 28/9/1979, O. L. Peixoto; EI 5604-05, 16/11/1979, O. L. Peixoto, E. Izecksohn; EI 5606-09, 13/12/1979, E. Izecksohn, O. L. Peixoto, M. C. A. Barbosa.

Remarks: EI 5604 now in MZUSP. Because of a possible printing mistake, the holotype of Hyla carvalhoi was cited as EI 6501 in the original publication. Actually EI 6501 is part of a series (EI 6501-EI 6503) of Leptodactylus jolyi specimens. The EI collection entry catalogue as well as the jar label designate specimen EI 5601 as the holotype of Hyla carvalhoi, so we call attention to that wrong information.

\section{Bokermannohyla gouveai (Peixoto \& Cruz, 1992): 197}

Original combination: Hyla gouveai Peixoto \& Cruz.

Type locality: Brejo da Lapa, Itatiaia, State of Rio de Janeiro, Brazil. $\left(22^{\circ} 23^{\prime} \mathrm{S}, 44^{\circ} 45^{\prime} \mathrm{W}\right)$, ca $1600 \mathrm{~m}$ elev.

Paratype: type locality, EI 8544, 28/11/1984, O. L. Peixoto, C. A. G. da Cruz.

Remarks: the elevation of the type locality is around $2100 \mathrm{~m}$, higher as stated in the original description.

Bokermannohyla izecksohni (Jim \& Caramaschi, 1979): 717

Original combination: Hyla izecksohni Jim \& Caramaschi.

Type locality: Rubião Junior, Botucatu, State of São Paulo, Brazil (22 $\left.53^{\circ} \mathrm{S}, 48^{\circ} 30^{\prime} \mathrm{W}\right)$, ca $800 \mathrm{~m}$ elev.

Paratype: type locality, EI 9358, 10/10/1968, T. C. F. Puppo, V. C. Jesus, ex JJ 1291.

\section{Dendropsophus pseudomeridianus (Cruz, Caramaschi \& Dias, 2000): 434}

Original combination: Hyla pseudomeridiana Cruz, Caramaschi \& Dias.

Type locality:, Seropédica, State of Rio de Janeiro, Brazil $\left(22^{\circ} 44^{\prime} \mathrm{S}\right.$, $\left.43^{\circ} 42^{\prime} \mathrm{W}\right)$, ca $40 \mathrm{~m}$ elev.

Paratype: type locality, EI 6251-6325, 11/1965, J. Jim, S. T. Albuquerque, A. Lebedenco, E. Izecksohn; EI 6417-6436, 10/ 1965, J. Jim, W. F. Mendonça, E. Izecksohn.
Dendropsophus ruschii (Weygoldt \& Peixoto, 1987): 238

Original combination: Hyla ruschii Weygoldt \& Peixoto.

Type locality: Domingos Martins, State of Espirito Santo, Brazil $\left(20^{\circ} 21^{\prime} \mathrm{S}, 40^{\circ} 39^{\prime} \mathrm{W}\right)$, ca $620 \mathrm{~m}$ elev.

Holotype: EI 7741, 12/1983, E. Izecksohn, C. A. G. da Cruz, S.

P. Carvalho e Silva, O. L. Peixoto.

Paratype: type locality, EI 7742, same data as holotype.

\section{Dendropsophus tritaeniatus (Bokermann, 1965): 259}

Original combination: Hyla tritaeniata Bokermann.

Type locality: São Vicente, Cuiabá, State of Mato Grosso, Brazil ( $\left.15^{\circ} 35^{\prime} \mathrm{S}, 56^{\circ} 05^{\prime} \mathrm{W}\right)$, ca $176 \mathrm{~m}$ elev.

Paratypes: type locality, EI 5641-42, 11/63, M. Alvarenga, F. M. Oliveira, W. C. A. Bokermann, ex WCAB 16213-12.

\section{Hypsiboas atlanticus (Caramaschi \& Vellosa, 1996): 2}

Original combination: Hyla atlantica Caramaschi \& Vellosa.

Type locality: CEPLAC, Itabuna, State of Bahia Brazil, (14 ${ }^{\circ} 55^{\prime} \mathrm{S}$, $\left.39^{\circ} 25^{\prime} \mathrm{W}\right)$, ca $54 \mathrm{~m}$ elev.

Paratypes: type locality, EI 8882-83, 12/1971, W. C.A. Bokermann.

\section{Hypsiboas latistriatus (Caramaschi \& Cruz, 2004): 62}

Original combination: Hyla latistriata Caramaschi \& Cruz. Type locality: Parque Nacional do Itatiaia, Brejo da Lapa, State of Minas Gerais, Brazil. (22 $\left.21^{\prime} \mathrm{S}, 44^{\circ} 44^{\prime} \mathrm{W}\right), 2160 \mathrm{~m}$ elev.

Paratypes: Marmelópolis, State of Minas Gerais, EI 3422-3426, 21/1/1965, J. Jim.

\section{Phyllodytes kautskyi Peixoto \& Cruz, 1988: 266}

Type locality: Domingos Martins, State of Espirito Santo, Brazil $\left(20^{\circ} 21^{\prime} \mathrm{S}, 40^{\circ} 39^{\prime} \mathrm{W}\right)$, ca $620 \mathrm{~m}$ elev.

Holotype: EI 7728, 8/1983, O. L. Peixoto, C. A. G. da Cruz, E. Izecksohn, S. P. Carvalho e Silva.

\section{Scinax agilis (Cruz \& Peixoto, 1982): 721}

Original combination: Ololygon agilis Cruz \& Peixoto.

Type locality: Ibiriba, Sooretama, State of Espírito Santo, Brazil (19²14'S, 3955'W), ca 20 m elev.

Holotype: EI 7123, 10/1979, E. Izecksohn, C. A. G. da Cruz, O. L. Peixoto.

Paratypes: Ponta da Fruta, Vila Velha, Estado do Santo, Brazil, EI 7124-38, 8/1973, O. L. Peixoto, C. A. G. da Cruz, E. Izecksohn; EI 7139-54, 7/1975, O. L. Peixoto, C. A. G. da Cruz, E. Izecksohn. Reserva Florestal Companhia Vale do Rio Doce, Sooretama, State of Espirito Santo, Brazil, EI 715556, 1/1980, S. P. Carvalho e Silva, C. A. G. da Cruz, J. S. Pinheiro, E. Izecksohn; EI 7157-67, 10/1980, E. Izecksohn, I. Ferreira, O. L. Peixoto.

Remarks: EI 7129 now in WCAB.

\section{Scinax cardosoi (Carvalho e Silva \& Peixoto, 1991): 264}

Original combination: Ololygon cardosoi Carvalho e Silva \& Peixoto.

Type locality: Vale da Revolta, Teresópolis, State of Rio de Janeiro, Brazil $\left(22^{\circ} 24^{\prime} \mathrm{S}, 42^{\circ} 57^{\prime} \mathrm{W}\right)$, ca $870 \mathrm{~m}$ elev. 
Paratype: km 13, Road Afonso Cláudio - Santa Maria, Domingos Martins, State of Espírito Santo, Brazil, EI 7752, 10/8/83, O. L. Peixoto, C. A. G. da Cruz, E. Izecksohn, S. P. Carvalho e Silva.

\section{Scinax heyeri (Peixoto \& Weygoldt, 1986): 432}

Original combination: Ololygon heyeri Peixoto \& Weygoldt.

Type locality: Santa Teresa, State of Espirito Santo, Brazil $\left(19^{\circ} 56^{\prime} \mathrm{S}, 40^{\circ} 36^{\prime} \mathrm{W}\right)$, ca $675 \mathrm{~m}$ elev.

Paralectotype: EI 7558, 4/8/1980, P. Weygoldt.

Remarks: As noted by Frost (2007), the Scinax heyeri description was amplified by Peixoto \& Weygoldt (1987), when a lectotype was designated from the syntype series.

\section{Scinax littoreus (Peixoto, 1988a): 257}

Original combination: Ololygon littorea Peixoto.

Type locality: Bambuí, Maricá, State of Rio de Janeiro, Brazil $\left(22^{\circ} 55^{\prime} \mathrm{S}, 42^{\circ} 49^{\prime} \mathrm{W}\right)$, sea level.

Holotype: EI 7562, 11/9/1980, E. Izecksohn, I. Ferreira, O. L. Peixoto.

Paratypes: type locality, EI 5221-29, 29/3/1975, O. L. Peixoto, C. A. G. da Cruz; EI 7563-69, same data as holotype; EI 7572-79, 17/ 7/1981, C. A. G. da Cruz, O. L. Peixoto. Arraial do Cabo, State of Rio de Janeiro, EI 3605-10, 10/5/1963, P. Juberg, H. Resende; EI 7570-71, 16/10/1983, E. Izecksohn, S. P. Carvalho e Silva.

Remarks: In the original description the paratypes series EI 522126 , was, in error, extended to include the EI numbers 5227, 5228 and 5229, but those numbers refer to part of a series of a Scinax cuspidatus sample and must be excluded from the paratypes series of Scinax littoreus. EI 7573 and EI 7577 now in AMNH.

\section{Scinax melloi (Peixoto, 1988b): 28}

Original combination: Ololygon melloi Peixoto.

Type locality: Parque Nacional da Serra dos Orgãos, Teresópolis, RJ, Brazil $\left(22^{\circ} 24^{\prime} \mathrm{S}, 42^{\circ} 57^{\prime} \mathrm{W}\right)$, ca $870 \mathrm{~m}$ elev.

Holotype: EI 7608, 15/10/1969, E. Izecksohn.

Paratypes: type locality, EI 869-72, 03/10/1958, R. P. Mello; EI 3560-69, 28-30/9/1962, E. Izecksohn, A. L. Peracchi; EI 357099, 2/1963, E. Izecksohn; EI 3600, 28/4/1964, E. Izecksohn; EI 5897-98, 1/4/1965, J. Jim, S. T. Albuquerque, E. Izecksohn, D. Moraes; EI 6945-49, 17/5/1966, E. Izecksohn, S. T. Albuquerque, A. Lebedenco, D. Moraes, EI 7609-13, 15/10/1969, E. Izecksohn, EI 7617, 1/1969, E. Izecksohn, EI 7618-27, 1/ 1970, C. A. G. da Cruz, EI 7715, 15/10/1969, E. Izecksohn.

Remarks: EI 7715 is listed in the original description as a paratype but is in reality one of the numbers in the type series of Euparkerella cochranae as seen in the entry of the EI collection. The actual number of the Scinax melloi specimen involved was changed to EI 7740, and this passed unnoticed.

\section{Scinax rizibilis (Bokermann, 1964): 430}

Original combination: Hyla rizibilis Bokermann.

Type locality: Campo Grande, Santo André, State of São Paulo, Brazil $\left(23^{\circ} 39^{\prime} \mathrm{S}, 46^{\circ} 32^{\prime} \mathrm{W}\right)$, ca $755 \mathrm{~m}$ elev.
Paratype: type locality, EI 5665, 20/10/1963, W. C. A. Bokermann, ex WCAB 14021.

\section{Sphaenorhynchus bromelicola Bokermann, 1966a: 18}

Type locality: Fazenda Santo Onofre, Maracás, Estado da Bahia, Brazil $\left(13^{\circ} 26^{\prime} \mathrm{S} 40^{\circ} 25^{\prime} \mathrm{W}\right)$, ca $960 \mathrm{~m}$ elev.

Paratype: type locality, EI 6117, 11/1965, F. M. Oliveira, W. C. A. Bokermann, ex WCAB 31942.

\section{Xenohyla truncata (Izecksohn, 1959): 259}

Original combination: Hyla truncata Izecksohn.

Type locality: km 37, Antiga Rodovia Presidente Dutra, Seropédica, State of Rio de Janeiro, Brazil $\left(22^{\circ} 44^{\prime} \mathrm{S} 43^{\circ} 43^{\prime} \mathrm{W}\right)$, ca $40 \mathrm{~m}$ elev.

Holotype: EI 107, 26/11/1958, S. G. Nunes, E. Izecksohn.

Paratypes: type locality, EI 101, 17/9/1958, B. A. M. Soares, G. R. Kloss, O. F. Fraga, E. Izecksohn, EI 102-105, 10/10/1958, O. F. Fraga, E. Izecksohn, EI 106, 108, and 109, same data as holotype.

Remarks: EI 102 now in WCAB (MZUSP).

\section{Hylidae: Phyllomedusinae Hylomantis granulosa (Cruz, 1988): 41}

Original combination: Phyllomedusa granulosa Cruz.

Type locality: Horto Zoobotânico Dois Irmãos, Recife, State of Pernambuco, Brazil $\left(08^{\circ} 03^{\prime} \mathrm{S}, 34^{\circ} 52^{\prime} \mathrm{W}\right)$, sea level.

Holotype: EI 7360, 7/1979, C. A. G. da Cruz, O. L. Peixoto, M. C. Barbosa.

Paratypes: type locality, EI 7358-59, EI 7361-62, same data as holotype.

\section{Phasmahyla exilis (Cruz, 1980): 683}

Original combination: Phyllomedusa exilis Cruz.

Type locality: Santa Teresa, State of Espirito Santo, Brazil (19 $\left.56^{\prime} \mathrm{S}, 40^{\circ} 36^{\prime} \mathrm{W}\right)$, ca $675 \mathrm{~m}$ elev.

Holotype: EI 5584, 31/3/1978, C. A. G. da Cruz, O. L. Peixoto, E. Izecksohn.

Paratypes: type locality, EI 5585, same data as holotype, EI 5586, 26/10/1974, C. A. G. da Cruz, S. T. Albuquerque, J. G. da Silva, E. Izecksohn.

\section{Phrynomedusa marginata (Izecksohn \& Cruz, 1976): 257}

Original combination: Phyllomedusa marginata Izecksohn \& Cruz. Type locality: Santa Teresa, State of Espírito Santo, Brazil $\left(19^{\circ} 56^{\prime} \mathrm{S}, 40^{\circ} 36^{\prime} \mathrm{W}\right)$, ca $675 \mathrm{~m}$ elev.

Holotype: EI 5177, 24/8/1974, S. T. Albuquerque, C. A. G. da Cruz, E. Izecksohn.

Paratypes: type locality, EI 5178, same data as holotype, EI 517986, 26/10/1974, J. G. da Silva, S. T. Albuquerque, C. A. G. da Cruz, E. Izecksohn, EI 5179, as allotype.

Remarks: EI 5186 now in JJ.

Phyllomedusa tetraploidea Pombal \& Haddad, 1992: 219

Type locality: Holambra II, Paranapanema, State of São Paulo, Brazil, $\left(23^{\circ} 23^{\prime} \mathrm{S}, 48^{\circ} 44^{\prime} \mathrm{W}\right)$, ca. $610 \mathrm{~m}$ elev. 
Paratypes: type locality, EI 8202-06, 23/2/1978, E. Izecksohn, C. Fröelich, J. Jim, C. A. G. da Cruz, O. L. Peixoto.

\section{Leptodactylidae}

Leptodactylus camaquara Sazima \& Bokermann, 1978: 907

Type locality: Conceição do Mato Dentro, Jaboticatubas, State

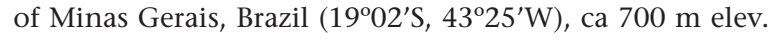

Paratype: type locality, EI 9360, 11/12/1974, W. C. A. Bokermann, I. Sazima, ex WCAB 48121.

Leptodactylus marambaiae Izecksohn, 1976b: 528

Type locality: Restinga da Marambaia, Rio de Janeiro, State of Rio de Janeiro, Brazil $\left(23^{\circ} 03^{\prime} \mathrm{S}, 4^{\circ} 38^{\prime} \mathrm{W}\right)$, sea level.

Holotype: EI 4123, 07/3/1969, J. Jim, S. T Albuquerque, C. A. G. da Cruz, E. Izecksohn.

Paratypes: type locality, EI 4115-17, 22/3/1968, C. Cupello, C. A. G. da Cruz, S. T. Albuquerque, E. Izecksohn; EI 4118-22, same data as holotype, EI 4122, as allotype.

Remarks: EI 4121 now in JJ.

Leptodactylus viridis Jim \& Spirandeli Cruz, 1979: 707

Type locality: Fazenda Pedra Branca, Itajibá, State of Bahia, Brazil

Paratype: Fazenda Barra do Cedro, Itajibá, State of Bahia, Brazil, EI 9361, 06/2/1974, U. Caramaschi, J. Jim, ex JJ 4527.

\section{Leiuperidae}

Physalaemus aguirrei Bokermann, 1966b: 194

Type locality: Floresta Nacional de Sooretama, Sooretama, State of Espirito Santo, Brazil (19²0'S, 39² $\left.40^{\prime} \mathrm{W}\right)$, sea level.

Paratype: type locality, EI 5746, 11/1964, W. C. A. Bokermann, ex WCAB 19321.

\section{Physalaemus centralis Bokermann, 1962: 216}

Type locality: Rio Coluene, Xingu, State of Mato Grosso, Brazil $\left(12^{\circ} 00^{\prime}, 53^{\circ} 22^{\prime} \mathrm{W}\right)$, ca $337 \mathrm{~m}$ elev.

Paratype: type locality, EI 2923, 3/11/1961, M. Alvarenga, W. C. A. Bokermann, ex WCAB 8124.

\section{Physalaemus cicada Bokermann, 1966c: 257}

Type locality: Fazenda Cana Brava, 10 km from Maracás, State of Bahia, Brazil $\left(13^{\circ} 27^{\prime} \mathrm{S}, 40^{\circ} 25^{\prime} \mathrm{W}\right)$, ca $1300 \mathrm{~m}$ elev.

Paratype: type locality, EI 6152, 11/1965, W. C. A. Bokermann, ex WCAB 32088.

\section{Physalaemus obtectus Bokermann, 1966b: 197}

Type locality: Floresta Nacional de Sooretama, Sooretama, State of Espirito Santo, Brazil. (19²0'S, 39² $\left.40^{\prime} \mathrm{W}\right)$, sea level.

Paratypes: type locality, EI 9466-67, 11/1964, W. C. A. Bokermann, ex WCAB 20574 and WCAB 20629.

\section{Physalaemus soaresi Izecksohn, 1965: 165}

Type locality: Floresta Nacional Mario Xavier, Seropédica, State of Rio de Janeiro, Brazil ( $\left.22^{\circ} 44^{\prime} \mathrm{S}, 43^{\circ} 43^{\prime} \mathrm{W}\right)$, ca $40 \mathrm{~m}$ elev.
Holotype: EI 1797, 2/1964, E. Izecksohn, J. Jim.

Paratypes: type locality, EI 1770-96, EI 1798, as allotype, 12/ 1962, E. Izecksohn; EI 1933-40, 11/1964, J. Jim, S. T. Albuquerque, W. F. Mendonça, E. Izecksohn; EI 1941-42, 12/1964, same collectors; EI 1943-45, 16/2/1965, S. T. Albuquerque, W. F. Mendonça, E. Izecksohn.

Remarks: EI 1796 now in JJ; EI 1936 now in WCAB.

\section{Hylodidae}

\section{Hylodes magalhaesi (Bokermann, 1964): 102}

Original combination: Elosia magalhaesi Bokermann.

Type locality: Campos do Jordão, State of São Paulo, Brazil $\left(22^{\circ} 44^{\prime} \mathrm{S}, 45^{\circ} 35^{\prime} \mathrm{W}\right)$, ca $1600 \mathrm{~m}$ elev.

Paratype: type locality, EI 2577, 12/1963, F. M. Oliveira, C. A. Campos Seabra, ex WCAB 14106.

Megaelosia lutzae Izecksohn \& Gouvêa, 1987: 17

Type locality: Maromba, Parque Nacional do Itatiaia, Resende, State of Rio de Janeiro, Brazil.

Holotype: EI 137, 20/11/1958, E. Izecksohn, E. Gouvêa.

\section{Cycloramphidae: Cycloramphinae} Crossodactylodes bokermanni Peixoto, 1982: 619

Type locality: Santa Teresa, State of Espirito Santo, Brazil, (1956's, 40³6’ W), ca $675 \mathrm{~m}$ elev.

Holotype: EI 7173, 22/12/1980, E. Izecksohn, P. Cascon, F. C. C. Rocha, O. L. Peixoto.

Paratypes: type locality; EI 7177, 31/4/1979, E. Izecksohn, O. L. Peixoto, C. A. G. da Cruz; EI 7178-79, 01/4/1979, E. Izecksohn, C. A. G. da Cruz, O. L. Peixoto; EI 7174-76, same data as holotype; EI 7180-83, 3/4/1981, C. A. G. da Cruz, E. Izecksohn, O. L. Peixoto, S. P. Carvalho e Silva, A. A. Reis; EI 7184-91, 29/8/1981, A. L. Carvalho, E. Izecksohn, C. A. G. da Cruz, O. L. Peixoto.

\section{Crossodactylodes izecksohni Peixoto, 1982: 621}

Type locality: Santa Teresa, State of Espirito Santo, Brazil, (195 $\left.56^{\prime} \mathrm{S}, 40^{\circ} 36^{\prime} \mathrm{W}\right)$, ca $675 \mathrm{~m}$ elev.

Holotype: EI 7192, 10/10/1980, E. Izecksohn, I. Ferreira, O. L. Peixoto.

Paratypes: type locality, EI 7193-222, same data as holotype; EI 7223-34, 29/8/1981, A. L. Carvalho, C. A. G. da Cruz, E. Izecksohn, O. L. Peixoto.

\section{Zachaenus carvalhoi Izecksohn, 1982: 7}

Type locality: Santa Teresa, State of Espirito Santo, Brazil (19 $\left.56^{\prime} \mathrm{S}, 40^{\circ} 36^{\prime} \mathrm{W}\right)$, ca $675 \mathrm{~m}$ elev.

Holotype: EI 7243, 28-29/10/1981, E. Izecksohn, C. A. G. da Cruz, O. L. Peixoto.

Paratypes: type locality, EI 7235, 11/3/1981, S. P. Carvalho e Silva, A. A. Reis, C. A. G da Cruz, E. Izecksohn; EI 7236-41, O. L. Peixoto, C. A. G. da Cruz, S. P. Carvalho e Silva, A. A. Reis, E. Izecksohn; EI 7242, EI 7244-52, same data as holotype.

Remarks: EI 7248 now in WCAB. 


\section{Cycloramphidae: Alsodinae}

Odontophrynus moratoi Jim \& Caramaschi, 1980: 357

Type locality: Rubião Junior, Botucatu, São Paulo, Brazil, (22 $\left.53^{\prime} \mathrm{S}, 48^{\circ} 30^{\prime} \mathrm{W}\right)$, ca $800 \mathrm{~m}$ elev.

Paratype: type locality, EI 9359, 17/10/1976, C. M. Carvalho, ex JJ 4553.

Proceratophrys laticeps Izecksohn \& Peixoto, 1981: 20

Type locality: Reserva Florestal da Companhia Vale do Rio Doce, Sooretama, State of Espirito Santo, Brazil. (19 $\left.14^{\prime} \mathrm{S}, 39^{\circ} 55^{\prime} \mathrm{W}\right)$, ca. $20 \mathrm{~m}$ elev.

Holotype: EI 5587, 01/11/1978, O. L. Peixoto.

Paratypes: type locality, EI 5588, 7/1978, S. T. Albuquerque, S. D. L. Raimundo, A. L. Peracchi; EI 5589, 9/1978, S. T. Albuquerque, S. D. L. Raimundo, A. Peracchi; EI 5590, 04/12/ 1978, C. A. G. da Cruz, O. L. Peixoto, S. P. Carvalho e Silva, E. Izecksohn; EI 5591-94, 1/5/1979, C. A. G. da Cruz, J. F. Pinheiro, O. L. Peixoto, E. Izecksohn.

Proceratophrys subguttata Izecksohn, Cruz \& Peixoto, 1998: 46 Type locality: Alto Palmeiras, Rio dos Cedros, State of Santa Catarina, Brazil $\left(26^{\circ} 44^{\prime} \mathrm{S}, 49^{\circ} 16^{\prime} \mathrm{W}\right)$, ca $850 \mathrm{~m}$ elev.

Holotype: EI 4770, 6/1966, L. W. Bernhardt, J. Jim.

\section{Bufonidae}

Dendrophryniscus berthalutzae Izecksohn, 1993b: 480

Type locality: Joinville, State of Santa Catarina, Brazil $\left(26^{\circ} 18^{\prime} \mathrm{S}\right.$, $\left.48^{\circ} 50^{\prime} \mathrm{W}\right)$, sea level.

Paratype: Alto Palmeiras, Rio dos Cedros, State of Santa Catarina, Brazil, EI 1968, 23/1/1964, L. W. Bernhardt.

\section{Dendrophryniscus bokermanni Izecksohn, 1993a: 407}

Type locality: Vila Amazonia, Parintins, State of Amazonas, Brazil ( $\left.2^{\circ} 37^{\prime} \mathrm{S}, 56^{\circ} 44^{\prime} \mathrm{W}\right)$, ca $27 \mathrm{~m}$ elev.

Holotype: EI 5399, 23/9/1972, J. A. Arrabal.

Paratypes: type locality, EI 5400-19, same data as holotype.

\section{Dendrophryniscus carvalhoi Izecksohn, 1993b: 478}

Type locality: Santa Teresa, State of Espírito Santo, Brazil $\left(19^{\circ} 56^{\prime} \mathrm{S}, 40^{\circ} 36^{\prime} \mathrm{W}\right)$, ca $675 \mathrm{~m}$ elev.

Holotype: EI 4127, 10/1968, A. Paviotti.

Paratypes: type locality, EI 4124-26, EI 4128, same data as holotype; EI 8877, 22/11/1980, O. L. Peixoto, P. Cascon, F. C. C. Rocha, E. Izecksohn; EI 8878, 05/2/1975, O. L. Peixoto.

\section{Dendrophryniscus leucomystax Izecksohn, 1968: 357}

Type locality: Reserva Biológica de Tingua, Nova Iguaçu, State of Rio de Janeiro, Brazil ( $\left.22^{\circ} 36^{\prime} \mathrm{S}, 43^{\circ} 26^{\prime} \mathrm{W}\right)$, ca $40 \mathrm{~m}$ elev.

Holotype: EI 4069, 10/1965, J. Jim, S. T. Albuquerque, W. F. de Mendonça, A. Lebedenco, E. Izecksohn.

Paratypes: type locality, EI 4045, 28/7/1965, J. Jim, W. F. de Mendonça, E. Izecksohn; EI 4046, 21/8/1965, J. Jim, S. T. Albuquerque, W. F. de Mendonça, E. Izecksohn; EI 4047-67, 9/1965, J. Jim, S. T. Albuquerque, W. F. de Mendonça, E.
Izecksohn; EI 4068-88, 10/1965, J. Jim, S. T. Albuquerque, A. Lebedenco, W. F. de Mendonça, E. Izecksohn, EI 4068, as allotype; 4089, 18/11/1965, J. Jim, S. T. Albuquerque, W. F. de Mendonça, A. Lebedenco, E. Izecksohn; EI 4090-4102, 12/1965, J. Jim, S. T. Albuquerque, W. F. de Mendonça, A. Lebedenco, E. Izecksohn; EI 4103-10, 09/11/1966, J. Jim, A. Lebedenco, E. Izecksohn.

Remarks: EI 4070-71 now in NMNH; EI 4085 now in JJ; EI 410102 now in $\mathrm{WCAB}$

\section{Microhylidae}

Chiasmodeis atlantica Cruz, Caramaschi \& Izecksohn, 1997: 59

Type locality: Reserva Biológica de Tinguá, Nova Iguaçu, State

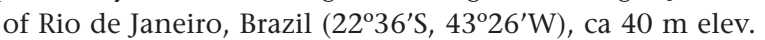

Paratypes: type locality, EI 8940-54, 27/12/1972, C. A. G. da Cruz, S. D. L. Raimundo, J. G. da Silva, E. Izecksohn.

Chiasmocleis capixaba Cruz, Caramaschi \& lzecksohn, 1997: 62 Type locality: Aracruz, State of Espírito Santo, Brazil, (1959'S, $\left.40^{\circ} 12^{\prime} \mathrm{W}\right)$, ca. $60 \mathrm{~m}$ elev.

Paratypes: type locality, EI 8955-56, 20-25/11/1994, R. P. Bastos, J. L. Gasparini, ex MNRJ 17530-31.

Chiasmocleis carvalhoi Cruz, Caramaschi \& Izecksohn, 1997: 65

Type locality: Floresta Nacional Mario Xavier, Seropédica, State of Rio de Janeiro, Brazil, (22 $\left.44^{\prime} \mathrm{S}, 43^{\circ} 43^{\prime} \mathrm{W}\right)$, ca $40 \mathrm{~m}$ elev.

Paratypes: type locality, EI 2096-235, 21/2/1964, J. Jim, E. Izecksohn; EI 2236-307, 11/1964, J. Jim, S. T. Albuquerque, W. F. de Mendonça, E. Izecksohn; EI 2308-99, 12/1964, J. Jim, S. T. Albuquerque, W. F. de Mendonça, E. Izecksohn; EI 4381-89, 12/1965, S. T. Albuquerque, W. F. de Mendonça, A. Lebedenco, E. Izecksohn, J. Jim: EI 4391-93, 19/10/1966, S. T. Albuquerque, W. F. de Mendonça, E. Izecksohn; EI 5332, 17/9/1975, E. Izecksohn, J. G. da Silva; EI 8937-38, 30/11/1979, I. Ferreira.

\section{Pipidae}

\section{Pipa arrabali Izecksohn, 1976a: 508}

Type locality: Vila Amazonia, Parintins, State of Amazonas, Brazil (2 $\left.37^{\circ} \mathrm{S}, 56^{\circ} 44^{\prime} \mathrm{W}\right)$, ca $27 \mathrm{~m}$ elev.

Holotype: EI 5311, 11/1972, J. A. Arrabal.

Remarks: Holotype destroyed by fungus, no remains.

\section{ACKNOWLEDGMENTS}

Stanley Rand and Ronald Heyer kindly reviewed drafts of the manuscript and encourage us to finish the present paper.

\section{REFERENCES}

Bokermann, W.C.A. 1962. Sobre uma pequena coleção de anfíbios do Brasil Central, com a descrição de uma espécie nova de Physalaemus (Amphibia, Salientia). Revista Brasileira de Biologia 22 (3): 213-219.

Bokermann, W.C.A. 1964. Uma nova espécie de Hyla da Serra do Mar em São Paulo (Amphibia, Salientia). Revista Brasileira 
de Biologia 24 (4): 429-434.

Bokermann, W.C.A. 1964. Una nueva espécie de Elosia de la Serra da Mantiqueira, Brasil. Neotropica 10 (33): 102-107.

BokermanN, W.C.A. 1965. Três novos batraquios da região central de Mato Grosso, Brasil. Revista Brasileira de Biologia 25 (3): 257-264.

Bokermann, W.C.A. 1966a. Duas novas espécies de Sphaenorhynchus (Amphibia, Hylidae). Revista Brasileira de Biologia 26 (1): 15-21.

Bokermann, W.C.A. 1966b. Dos nuevas espécies de Physalaemus de Espirito Santo, Brasil (Amphibia, Leptodactylidae). Physis 26 (71): 193-202.

Bokermann, W.C.A. 1966c. Notas sôbre três espécies de Physalaemus de Maracás, Bahia (Amphibia, Leptodactylidae). Revista Brasileira de Biologia 26 (3): 253-259.

Caramaschi, U. \& A. Velosa. 1996. Nova espécie de Hyla Laurenti, 1768 do Leste Brasileiro (Amphibia, Anura, Hylidae). Boletim do Museu Nacional, Nova Série Zoologia (365): 1-7.

Caramaschi, U. \& C.A.G. Cruz. 2004. Duas novas espécies de Hyla do grupo de H. polytaenia Cope, 1870 do Sudeste do Brasil (Amphibia, Anura, Hylidae). Arquivos do Museu Nacional 62 (3): 247-254.

Carvalho e Silva, S.P. de \& O.L. Рeixoto. 1991. Duas novas espécies de Ololygon para os Estados do Rio de Janeiro e Espírito Santo (Amphibia, Anura, Hylidae). Revista Brasileira de Biologia 51 (1): 263-270.

Cruz, C.A.G. DA. 1980. Descrição de uma nova espécie de Phyllomedusinae do Estado do Espirito Santo (Amphibia, Anura, Hylidae). Revista Brasileira de Biologia 40 (4): 683-687.

Cruz, C.A.G. DA. 1988 [1989]. Sobre Phyllomedusa aspera e a descrição de uma espécie nova desse gênero (Amphibia, Anura, Hylidae). Arquivos da Universidade Federal Rural do Rio de Janeiro 11 (1-2): 39-44.

Cruz, C.A.G. DA \& O.L. Peixoto. 1982. Uma nova espécie de Hyla do Estado do Espirito Santo, Brasil (Amphibia: Anura: Hylidae). Revista Brasileira de Biologia 42 (4): 721-724.

Cruz, C.A.G. DA \& O.L. Реixoto. 1984. Espécies verdes de Hyla: o complexo "albosignata" (Amphibia, Anura, Hylidae). Arquivos da Universidade Federal Rural do Rio de Janeiro 7 (1): 31-47.

Cruz, C.A.G. da \& O.L. Peixoto. 1985 [1987]. Espécies verdes de Hyla: o complex "albofrenata" (Amphibia, Anura, Hylidae). Arquivos da Universidade Federal Rural do Rio de Janeiro $8(1-2): 59-70$.

Cruz, C.A.G. DA \& O.L. Peixoto. 1998. Informar a referência completa seguindo as Instruções aos Autores.

Cruz, C.A.G.da; U. Caramaschi \& E. Izecksohn. 1997. The genus Chiasmocleis Méhely, 1904 (Anura, Microhylidae) in the Atlantic rain forest of Brazil, with description of three new species. Alytes 15 (2): 49-71.

Cruz, C.A.G.da; U. Caramaschi \& A.G. Dias. 2000. Espécie nova de Hyla Laurenti, 1768 do Estado do Rio de Janeiro, Brasil. Boletim do Museu Nacional, Nova Série, Zoologia (434):
$1-8$.

Faivovich, J.; P.C.A. Garcia; C.F.B. Haddad; D.R. Frost; J.A Campbell $\&$ W.C. Wheeler. 2005. Systematic Review of the Frog Family Hylidae, With Special Reference to Hylinae: Phylogenetic Analysis and Taxonomic Revision. Bulletin American Museum of Natural History 294: 1-240.

Frost. 2007. Amphibian species of the world: an online reference. V.5.0. Available in the Wide World Web at http:/ /research.amnh.org/herpetology/amphibia/index.html [accessed in 15/VI/2007].

Frost, D.R.; T. Grant; J. Faivovich; R.H. Bain; A. HaAs; C.F.B. Haddad; R.O. de SÁ; A.Channing; M.Wilkinson; S.C Donnellan; C.J. Raxworthy; J.A. Campbell; B.L Blotto; P. Moler; R.C. Drewes; R.A. Nusbaum; J.D. Lynch; D.M. Green \& W.C. Wheeler. 2006. The Amphibian Tree of Life. Bulletin American Museum of Natural History 297: 1-370.

Grant, T; D.R. Frost; J.A Campbell; R. Gagliardo; C.F.B. Haddad; P.J.R. KoK; D.B. Means; B.P. NoOnan; W.E. Schargel \& W.C. Wheeler. 2006. Phylogenetic Systematics of Dart Poison Frogs and Their Relatives (Amphibia: Athesphatanura: Dendrobatidae). Bulletin American Museum of Natural History 299: 1-262.

Heyer, R. 1984. Variation, systematics, and zoogeography of Eleutherodactylus guentheri and closely related species (Amphibia, Anura, Leptodactylidae). Smithsonian Contributions to Zoology (402): 1-42.

ICZN. 1999. Internacional Code of Zoological Nomenclature. London, International Trust for Zoological Nomenclature, $4^{\text {th }}$ ed., 338p.

Izecksohn, E. 1959. Uma nova espécie de Hylidae da Baixada Fluminense, Estado do Rio de Janeiro, Brasil. Revista Brasileira de Biologia 19 (3): 259-263.

Izecksohn, E. 1965. Uma nova espécie de Physalaemus Fitzinger, do Estado do Rio de Janeiro (Amphibia, Anura). Revista Brasileira de Biologia 25 (2): 165-168.

Izecкsonn, E. 1968. Nova espécie de Dendrophryniscus do Estado do Rio de Janeiro (Amphibia, Salientia). Revista Brasileira de Biologia 28 (4): 357-362.

Izecksohn, E. 1971. Novo gênero e nova espécie de Brachycephalidae do Estado do Rio de Janeiro, Brasil (Amphibia, Anura). Boletim do Museu Nacional do Rio de Janeiro, Nova Série Zoologia, 280: 1-12.

Izecкsonn, E. 1976a. Uma nova espécie de Pipa, do Estado do Amazonas, Brasil (Amphibia, Anura, Pipidae). Revista Brasileira de Biologia 36 (2): 507-510.

Izeскsонn, E. 1976b. Uma nova espécie de Leptodactylus, do Estado do Rio de Janeiro, Brasil (Amphibia, Anura, Leptodactylidae). Revista Brasileira de Biologia 36 (2): 527-530.

Izecksohn, E. 1982. Uma nova espécie de Zachaenus Cope, do Estado do Espirito Santo, Brasil (Amphibia, Anura, Leptodactylidae). Arquivos da Universidade Federal Rural do Rio de Janeiro 5 (1): 7-11.

IzECKSOHN, E. 1988. Algumas considerações sobre o gênero Eupar- 
kerella, com a descrição de três novas espécies (Amphibia, Anura, Leptodactylidae). Revista Brasileira de Biologia 48 (1): 59-74.

Izecksohn, E. 1993a. Nova espécie de Dendrophryniscus da região amazônica (Amphibia, Anura, Bufonidae). Revista Brasileira de Biologia 10 (3): 407-412.

Izecksonn, E. 1993b. Três novas espécies de Dendrophryniscus Jimenez de la Espada das regiões sudeste e sul do Brasil (Amphibia, Anura, Bufonidae). Revista Brasileira de Biologia 10 (3): 473-488.

Izecksohn, E. \& C.A.G.DA Cruz. 1976. Nova espécie de Phyllomedusinae do Estado do Espirito Santo, Brasil (Amphibia, Anura, Hylidae). Revista Brasileira de Biologia 36 (1): 257-261.

Izecrsohn, E. \& E. GouvêA. 1985 [1987]. Nova espécie de Megaelosia, de Itatiaia, Estado do Rio de Janeiro (Amphibia, Anura, Leptodactylidae). Arquivos da Universidade Federal Rural do Rio de Janeiro 8 (1-2): 17-22.

Izecksonn, E. \& O.L. Peixoto. 1981. Nova espécie de Proceratophrys, da hiléia Bahiana, Brasil (Amphibia, Anura, Leptodactylidae). Revista Brasileira de Biologia 41 (1): 19-24.

Izecrsonn, E. \& O.L. Pеiхото. 1998. Sobre Proceratophrys appendiculata e algumas espécies afins (Amphibia, Anura, Leptodactylidae). Revista da Universidade Rural, Série Ciências da Vida, 20 (1-2): 37-54.

Jim, J. \& U. Caramaschi. 1979. Uma nova espécie de Hyla da região de Botucatu, São Paulo, Brasil (Amphibia, Anura). Revista Brasileira de Biologia 39 (3): 717-719.

Jim, J. \& E.F.Spirandeli Cruz. 1979. Uma nova espécie de Leptodactylus do Estado da Bahia, Brasil (Amphibia, Anura). Revista Brasileira de Biologia 39 (3): 707-710.

Jim, J. \& U. Caramaschi. 1980. Uma nova espécie de Odontophrynus da região de Botucatu, São Paulo, Brasil (Amphibia, Anura). Revista Brasileira de Biologia 40 (2): 357-360.

Levington, A.E.; R.H. Gibbs Jr; E. Heal, C. Jim, J. \& E. Dawson. 1985. Standards in Herpetology and Ichthyology: Part I. Standard Symbolic Codes for Institutional Resource Collections in Herpetology and Ichthyology. Copeia 1985 (3): 802-832.

Peixoto, O.L. 1981. Nova espécie de Hyla da Serra dos Orgãos, Estado do Rio de Janeiro, Brasil (Amphibia: Anura: Hylidae). Revista Brasileira de Biologia 41 (3): 515-520.

Peixoto, O.L. 1982. Duas novas espécies de Crossodactylodes de Santa Tereza, Estado do Espírito Santo, Brasil (Amphibia,
Anura, Leptodactylidae). Revista Brasileira de Biologia 42 (3): 619-626.

Peixoto, O.L. 1988a. Sobre o "status" taxonomico de Hyla catharinae alcatraz B. Lutz 1973, com a descrição de uma nova espécie para o grupo "perpusilla" (Amphibia, Anura, Hylidae). Acta Biologica Leopoldensia 10 (2): 253-267.

Peixoto, O.L. 1988b [1989]. Duas novas espécies de Ololygon do grupo 'perpusilla' (Amphibia, Anura, Hylidae). Arquivos da Universidade Federal Rural do Rio de Janeiro 11 (1-2): 27-37.

Peixoto, O.L. \& C.A.G. DA Cruz. 1988. Descrição de duas espécies novas do gênero Phyllodytes Wagler (Amphibia: Anura: Hylidae). Revista Brasileira de Biologia 48 (2): 265-272.

Peixoto, O.L. \& C.A.G.DA Cruz. 1992. New species of Hyla from the "Serra da Mantiqueira, Itatiaia, Rio de Janeiro" state (Amphibia, Anura, Hylidae). Memórias do Instituto Oswaldo Cruz 87 (Suppl. 1): 197-200.

Peixoto, O.L. \& E. Izecksohn. 2001. A coleção herpetológica Eugenio Izecksohn. Contribuições Avulsas sobre a História Natural do Brazil, Série Zoologia, 31: 1-4.

Peixoto, O.L. \& P. Weygoldt. 1986. Informar a referência completa seguindo as Instruções aos Autores.

Peixoto, O.L. \& P. Weygoldt. 1987. Notes on Ololygon heyeri Weygoldt, 1986 from Espirito Santo, Brazil (Amphibia: Salientia: Hylidae). Senckenbergiana Biologica 68 (1-3): 1-9.

Pombal JR, J.P. \& C.F.B. Haddad. 1992. Espécies de Phyllomedusa do grupo burmeisteri do Brasil oriental, com descrição de uma espécie nova (Amphibia, Hylidae). Revista Brasileira de Biologia 52 (2): 217-229.

Sazima, I. \& W.C.A. Bokermann. 1978. Cinco novas espécies de Leptodactylus do centro e sudeste Brasileiro (Amphibia, Anura, Leptodactylidae). Revista Brasileira de Biologia 38 (4): 899-912.

Weygoldt, P. 1986. Beobachtungen zur ökologie und Biologie Von Froschen an einem neotropischen Bergbach. Zoolisch Jahrbücher Systematics 113 (3): 429-454.

Weygoldt, P. \& O.L. Peixoto. 1987. Hyla ruschii sp. nov., a new frog from the Atlantic Forest Domain in the State of Espirito Santo, Brazil (Amphibia, Hylidae). Studies on Neotropical Fauna and Environment, 22 (4): 237-247.

Wiley, E.O. 1981. Phylogenetics: the theory and practice of phylogenetic systematics. New York, John Wiley and Sons, 439p.

Received in 01.XII.2006; accepted in 20.VIII.2007.

Revista Brasileira de Zoologia 24 (3): 721-728, setembro 2007 